VALORACIÓN DEL CONSUMIDOR DEL NORDESTE DE MÉXICO HACIA LAS INNOVACIONES EN EL YOGUR ${ }^{1}$

\title{
VALUATION OF THE CONSUMER OF THE NORTHWESTERN MEXICO TOWARDS INNOVATIONS IN THE YOGURT
}

\author{
Dena $\mathrm{M}^{\mathrm{a}}$ Camarena Gómez ${ }^{2}$, Lizbeth Salgado Beltrán ${ }^{3}$, Luis Antonio Terán ${ }^{4}$, \\ Ramo Barrena ${ }^{5}$ y Alejandro Medellín Palencia ${ }^{6}$. Universidad de Sonora. Hermosillo-México
}

RECIBIDO: Agosto 25 de 2014

ACEPTADO: Noviembre 13 de 2014

\section{RESUMEN}

En esta investigación, se analizó la valoración que realiza el consumidor del noroeste de México hacia las innovaciones del yogur. Se seleccionó este producto por ser uno de los de mayor disponibilidad en el mercado, conocido por una gran proporción de consumidores y por presentar un importante número de innovaciones. Se utilizó el enfoque cuantitativo, con un método exploratorio, a través de un diseño transversal. Se emplearon dos herramientas; la primera fueron recorridos (24), realizados en diferentes establecimientos comerciales y la segunda fueron encuestas (400), aplicadas a consumidores mayores de 18 años. Se hicieron análisis descriptivos (media y moda), así como bivariantes (prueba de anova y test de chi-cuadrado) y multivariados (análisis factorial y de conglomerado). Los resultados muestran que se comercializa en mayor medida el yogur tradicional, mientras que los funcionales tienen un menor grado de penetración en el mercado. Se trata principalmente de productos con innovaciones incrementales. Se encontró que existen dos grupos de consumidores: el primero se orienta hacia innovaciones en el diseño y la forma de consumo, mientras que el segundo se enfoca en innovaciones de sabor y seguridad. En el primero, existe una mayor proporción de consumidores de 33 a 47 años de edad, en tanto que los mayores de 47 años se orientan más hacia la seguridad. También se encontró que el nivel de estudios influye en la valoración de atributos del yogur.

Palabras clave: análisis cuantitativo [172], comportamiento innovador [81], consumidor [78], evaluación [1436], alimento [69].

\begin{abstract}
In this research, it was analyzed the valuation made by the consumer of northwestern of Mexico towards yogurt innovations . This product was selected, because it's one of the most widely available in the market, known by a large proportion of consumers and because it presents a significant number of innovations. It was used a quantitative approach, with an exploratory method, trough a transversal design. It were employed two tools, the first one were linear routes, making 24 in different commercial establishments and the second one were surveys, applying 400 surveys to consumers older than 18 years. Analyzis carried out were descriptive (mean and mode), bivariate (Anova Test and Chi-square Test) and multivariate (factor analysis and a cluster). The results showed that traditional yogurt is a product well established in the market, while the functional one has a lower degree of penetration in it. All of them are known for having incremental innovations. It was found that there are two consumer groups, the first, focuses on innovations in design and way of consumption, it's composed mostly by men in the range of 33-47 years, while the second, focuses on taste and safety innovations. In the first, there is a greater proportion of consumers between 33-47 years of age, while those over 47 years are more oriented towards safety. It has been also found that the level of education affects the value of attributes of yogurt.
\end{abstract}

Key words: Quantitative Behavior [108], Consumer [78], Evaluation [1436], Innovative Behaviour [81], Food [69].

Este Artículo se puede referenciar

Camarena, D., Salgado, L., Terán, L., Barrena, M. \& Medellín, A. (2015). Valoración del consumidor del noroeste de México hacia las innovaciones en el yogur, en Desarrollo Gerencial Revista de la Facultad de Ciencias Económicas, administrativas y contables de la Universidad Simón Bolívar 7(1), 16-29.

\footnotetext{
${ }^{1}$ Proyecto de Investigación. Condicionantes psicográficos y socioeconómicos que influyen en la aceptación de innovaciones alimentarias: un análisis cross-cultural entre México y España (PROMEP/103.5/13/6832).

2 Doctora. Profesora-Investigadora en el Departamento de Contabilidad, Campus Hermosillo de la Universidad de Sonora. dena.camarena@eca.uson.mx

${ }^{3}$ Doctora. Profesora- Investigadora en el Campus Caborca de la Universidad de Sonora. Isalgado@caborca.uson.mx

${ }^{4}$ Pasante de Licenciatura. Tesista en el Departamento de Economía, Campus Hermosillo de la Universidad de Sonora. l-teran@hotmail.com

5 Doctora. Profesora-Investigadora en el Departamento de Gestión de Empresas de la Universidad Pública de Navarra. ramo.barrena@unavarra.es

6 Doctor. Profesor Investigador en el Departamento de Contabilidad, Campus Hermosillo de la Universidad de Sonora. alejandromp@pitic.uson.mx
}

Desarrollo Gerencial, 7 (1) pp. 16 - 29 Enero-Junio 2015. ISSN: 2145-5147 (On Line). Universidad Simón Bolívar. Barranquilla-Colombia. Contactos: desarrollogerencial@unisimonbolivar.edu.co 


\section{1.- INTRODUCCIÓN}

Antes del proceso globalizador las economías y las actividades empresariales, que en otros periodos de tiempo solo se veían influenciadas por elementos internos de la industria o de índole nacional, hoy cada vez tienen mayor interrelación debido a factores como el comercio internacional, el desarrollo de los medios de comunicación, el ensamblado y producción en distintos puntos geográficos, entre otros. Las implicaciones empresariales de estos elementos permiten, por un lado, un mayor acceso a los mercados internacionales y, por otro, una mayor y más intensa concurrencia competitiva.

En el ámbito de las empresas alimentarias, esta situación condiciona su desarrollo y presiona hacia la competitividad, la cual lleva a invertir una gran cantidad de recursos en la búsqueda de una innovación que revolucione el mercado o que mejore los productos ya existentes (Hoban, 1998). Pese a esta presión por competir e innovar, si se compara el sector agroalimentario con otros, como el electrónico y biotecnológico, se advierte un bajo nivel de innovación y de orientación al mercado (Winger \&Wall, 2006; Arcas, 2003). Esta tendencia aumenta cuando se considera que el 75\% de los bienes introducidos en el mercado son extensiones de líneas de productos, también conocidas como innovaciones continuas.

No obstante, hay que tener en cuenta que esta proporción puede variar de un país a otro, tal es el caso de Tailandia donde un $9 \%$ de los productos alimentarios que son introducidos al mercado se pueden clasificar como innovadores, mientras que un $40 \%$ se catalogan como extensiones de líneas de producto (Siriwongwilaichat, 2001). Esto lleva a constatar que las cifras de innovaciones, sean estas continuas o radicales, no son homogéneas entre países. Pese a ello se ha estimado de manera general un ratio de fracaso en la industria de la alimentación, el cual oscila entre 75 y 80\% (Barrena, García, \& LópezMosquera, 2013; Winger \&Wall, 2006).

En este contexto, vale la pena reflexionar sobre los factores que intervienen en el éxito o fracaso de un producto, los cuales en gran medida pueden estar condicionados por aspectos económicos, sociales y culturales. Es así que el cambio o introducción de un nuevo producto puede ser rechazado en el mercado porque no cumple las expectativas del consumidor, por la resistencia hacia lo nuevo, el arraigo hacia lo tradicional, a lo que ya se conoce y resulta familiar, entre otros.

En esta línea, algunos autores reconocen la importancia de la demanda final en el proceso de aceptación o rechazo de las innovaciones. De ahí que para mantenerse competitivos consideran necesarias la innovación y la producción con una orientación hacia el mercado. Si bien existen otros factores que también influyen en el proceso, como son la educación y los medios de comunicación, lo cierto es que la decisión final recae en el consumidor (Earle, 1997). 


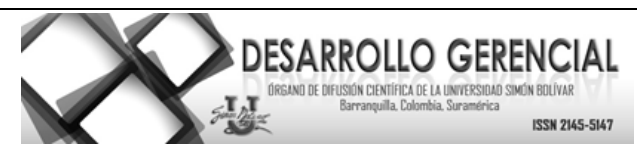

Desde esta perspectiva los estudios de las innovaciones alimentarias centrados en el consumidor son una constante en la investigación, aun cuando en el noroeste de México todavía son escasos. De ahí que esta aportación permite ahondar en el conocimiento y preferencias del consumidor del estado Sonora (ubicado al noroeste de México), segundo en extensión territorial en el país $\left(179,502.89 \mathrm{~km}^{2}\right.$ ) y con una población de 2'662,480 habitantes. Así, el objetivo principal de esta investigación es analizar la valoración que realizan los consumidores sonorenses hacia las innovaciones en un producto alimentario tradicional como el yogur. La selección de este producto obedece a sus características de consumo, ya que forma parte de la dieta habitual de personas de distintos estratos socioeconómicos, es conocido por una amplia proporción de la población, y desde su surgimiento hasta la actualidad ha presentado un significativo número de innovaciones. Desde el punto de vista comercial se caracteriza por un fácil consumo, un precio asequible y disponibilidad en un amplio número de establecimientos (Briones, 2005; Salcedo, Font \& Martínez, 1998).

\section{2.- MÉTODO}

\section{Diseño}

Este estudio es una primera aproximación de las valoraciones que realiza el consumidor del noroeste de México hacia las innovaciones alimentarias; por ello su análisis se realiza desde un enfoque cuantitativo, utilizando el método exploratorio, con un diseño transversal, tal y como se sugiere en la literatura (Hernández, Fernández \& Baptista, 2010).

Las herramientas utilizadas en la investigación fueron dos: la primera consistió en la obtención directa de información a través de los recorridos de lineales/anaqueles en las cadenas de distribución, y la segunda aplicó encuestas dirigidas a los consumidores. Una vez concluido el trabajo de campo se procedió a su procesamiento conforme los cuatro pasos sugeridos por Hernández et al. (2010): 1) codificar las categorías de ítems, preguntas y categorías de contenido u observaciones no precodificadas; 2) elaborar el libro de códigos; 3) efectuar físicamente la codificación y 4) grabar y guardar los datos en un archivo permanente. Así, después de generar los archivos con los datos codificados en valores numéricos, se abocó a su análisis mediante el programa IBM SPSS 20.0.

Posteriormente se procedió a realizar los diferentes tipos de análisis. Los métodos utilizados fueron tres: el método de análisis univariante, el análisis bivariante y el multivariante. En el método univariante se efectuaron análisis de frecuencia y descriptivos (medias y modas); en los análisis bivariantes se realizaron tests de chi-cuadrado (de Pearson) y la prueba de Anova o test de comparación de medias; 


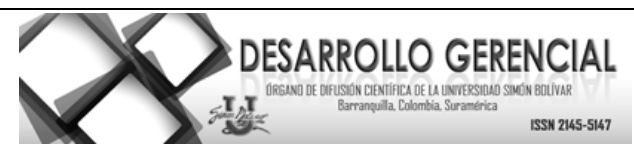

mientras que en el método multivariante se llevaron a cabo análisis factoriales exploratorios, aplicándose los tests de fiabilidad (alpha de Cronbach), la medida de adecuación muestral de Kaiser-Meyer-Olkin (KMO) y la prueba de esfericidad de Bartlett. Dentro de los métodos multivariante se realizó un análisis clúster o de conglomerados. Estas técnicas de análisis son de uso habitual en distintas investigaciones donde se busca establecer las características y perfil del consumidor (Díaz, Prieto \& Bernabéu, 2013; Camarena, Sanjuán \& Philippidis, 2011; Montoya, 2007; Fernández, 2004; Ritchey, 2002, Díaz de Rada, 1998.

\section{Participantes}

Las personas que colaboraron en la investigación son habitantes de la ciudad de Hermosillo, que cuenta con 510,564 individuos mayores de 18 años de edad (Inegi, 2010). Se realizó un muestreo probabilístico aleatorio simple con afijación proporcional por edad y sexo, con un error muestral del 5\% y un nivel de confianza del 95.5\%; el resultado arrojó una muestra de 400 individuos.

\section{Instrumentos}

Los instrumentos utilizados son dos: los recorridos de lineales y las encuestas personales. Se trata de herramientas ampliamente utilizadas en investigaciones de mercado donde se busca conocer las características de la oferta, así como preferencias y valoraciones de los consumidores (Ramírez \& Jiménez, 2013; Alvarado, Luyando \& Téllez, 2012; Cortés, Mora, García-Mata \& Ramírez-Valverde, 2012; Camarena \& Sanjuán, 2006.

El primer instrumento utilizado permite obtener datos relativos a las características que tiene el producto al momento de llegar al consumidor final. El formato de recolección de información contenía siete variables: una relativa a los aspectos nutrimentales, el peso, el precio, el tipo de envase, la marca, la distribución en el establecimiento comercial y la fecha de caducidad. El propósito de reunir esta información directamente de los puntos de venta fue conocer los productos que se encuentran en el mercado, además de obtener una descripción detallada de sus características y las estrategias de ubicación utilizadas en los establecimientos. Para seleccionar los establecimientos, primero se procedió a clasificarlos de acuerdo a los servicios que ofrecen y a las características de comercialización; así se organizaron en: hipermercado, supermercado, bodega, tienda de conveniencia y clubes de membrecía. El criterio de selección fue la ubicación geográfica en la ciudad, buscando representatividad de todo el entorno urbano, además de incluir al menos una sucursal de aquellas empresas con mayor cuota de 


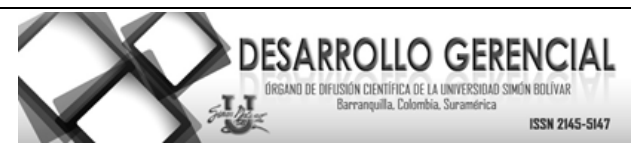

mercado en la región. Así, se realizaron recorridos de lineales en 24 establecimientos comerciales de la ciudad de Hermosillo (México) durante el mes de abril del 2014.

El segundo instrumento hace posible conocer las características de comportamiento que tiene el consumidor del noroeste de México (Hermosillo) al momento de comprar y consumir yogur. Se realizó un cuestionario estructurado en cuatro secciones denominadas: hábitos de compra, hábitos de consumo, preferencias y características sociodemográficas. En total, el cuestionario está integrado por 19 preguntas de tipo abierto, cerrado, de respuesta múltiple, de escala y de elección de preferencias. Previamente se realizaron pruebas pilotos con el fin de corregir y minimizar errores antes de su aplicación definitiva. El trabajo de campo se realizó entre los meses de mayo y junio de 2014, en la ciudad de Hermosillo (México). Se aplicó a personas mayores de 18 años de edad, y los que participaron lo hicieron sin recibir ningún tipo de remuneración económica, fueron seleccionadas al azar en sitios públicos, en lugares de ocio y esparcimiento como plazas y parques, así como en las inmediaciones de lugares de reunión multitudinarios como iglesias y centros comerciales. Los resultados que se presentan corresponden a aquellas variables que permiten alcanzar el objetivo planteado en esta investigación.

\section{Procedimientos}

La investigación se compone de cinco fases. La primera llevó a una revisión de literatura acerca de innovaciones y del sector agroalimentario. En la segunda fase se visitaron los principales establecimientos comerciales de la ciudad, con el fin de definir el producto que sería objeto de estudio, y se encontraron cuatro que cumplían con el condicionante de mostrar innovaciones en su envase. Sin embargo, se seleccionó el yogur por ser el artículo más conocido por los consumidores, el de mayor disponibilidad y por estar disponible en el mismo formato en los distintos establecimientos y presentar distintos grados de innovación. La tercera fase se llevó a cabo en el mes de abril de 2014, y consistió en realizar recorridos de lineales en los principales centros comerciales de autoservicio para conocer las características de la oferta de yogur. La cuarta fase consistió en la elaboración y aplicación de la encuesta durante mayo y junio de 2014. La última fase, la quinta, correspondió al análisis de la información y posterior redacción de resultados.

\section{3.- RESULTADOS Y DISCUSIÒN}

Los resultados permiten conocer la valoración del consumidor del noroeste de México hacia las innovaciones en el yogur. De acuerdo a lo obtenido, se identificó que de los 24 establecimientos visitados y donde se efectuaron los recorridos de lineales, el 100\% comercializa yogur con marca, siendo la más 


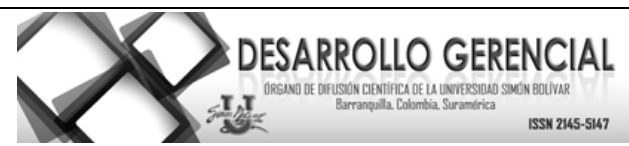

frecuente Danone (35.8\%), seguida de Yoplait (31.9\%) y Lala (21.2\%). Otras como Nestlé, Yaqui, Chilchota, Nutriyogurth y Yogufrut se encuentran en el 10.9\% de los establecimientos.

Considerando el tipo de yogur, se observa que el normal/tradicional (70.5\%) es el que se comercializa en un mayor número de establecimientos, seguidos de los denominados probióticos (12\%) y los de estilo griego (9.5\%). Otras modalidades (7.8\%) también se encuentran disponibles, pero en un menor número de negocios (tabla 1).

Tabla 1. Tipos de yogur de acuerdo a su disponibilidad de comercialización

\begin{tabular}{|l|c|}
\hline \multicolumn{1}{|c|}{ Tipo de yogur } & $\begin{array}{c}\text { Establecimientos que lo } \\
\text { comercializan (\%) }\end{array}$ \\
\hline Tradicional (de sabores) & 70.5 \\
\hline Probióticos & 12 \\
\hline Griego & 9.5 \\
\hline Otros (deslactosado, natural y cremoso) & 7.8 \\
\hline
\end{tabular}

Fuente: elaboración propia.

De acuerdo al embalaje de los productos, el 75.3\% de los yogures disponibles presentan diseños innovadores, siendo la botella con diseño la de mayor disponibilidad (56.0\%), seguida del vaso y charola con diseño, con un 12.7 y $6.7 \%$, respectivamente. Otras opciones, con diseño sencillo o tradicional, también se encuentran disponibles en el mercado, aunque en menor medida, como es el caso del vaso sencillo (16.7\%), la charola (4.6\%), botella (1.7\%) y bote sencillo (1.4\%), (tabla 2). Esta disponibilidad muestra que las innovaciones en el envase se encuentran presentes en la oferta de yogures, posicionándose como una estrategia de uso frecuente por los empresarios.

Tabla 2. Disponibilidad de yogur de acuerdo a su envase y diseño

\begin{tabular}{|l|c|}
\hline \multicolumn{1}{|c|}{ Diseño } & Oferta (\%) \\
\hline Botella con diseño & 56.0 \\
\hline Vaso con diseño & 12.7 \\
\hline Charola con diseño & 6.7 \\
\hline Vaso sencillo & 16.7 \\
\hline Charola sencilla & 4.6 \\
\hline Botella sencilla & 1.7 \\
\hline Bote sencillo & 1.4 \\
\hline
\end{tabular}

Fuente: elaboración propia.

Asimismo, se encontró que la información disponible en la etiqueta cumple con los dispuesto en las normas oficiales mexicanas, NOM-181-SCFI-2010 y la NOM-051-SCFI-2010, en donde se indica que el yogur al igual que otros productos alimenticios debe llevar inscrito en su envase la información comercial, 
la fecha de caducidad o la fecha de consumo preferente, el lugar de envasado y el país de origen de la mercancía, todo escrito en idioma español. Se advierte que el 100\% de los productos analizados incluyen esta información y cumplen con la normativa.

Con respecto al precio no existe uniformidad, ya que este varía en función de la cantidad y el tipo de yogur. El precio mínimo es de $\$ 2.75$ y puede llegar hasta los $\$ 11.10$ pesos. Estas variaciones se asocian a las ofertas que se realizan, pero también a la ubicación geográfica, ya que establecimientos de la misma cadena comercial ofrecen precios distintos según su ubicación y el estrato socioeconómico de su zona de influencia.

En cuanto a los gustos y preferencias de los consumidores, se orientan en mayor medida hacia el yogur natural (46.0\%), seguido del yogur líquido $(38.0 \%)$ y el yogur batido $(13.9 \%)$, mientras que una menor proporción elige el que tiene efecto bífidus (2.1\%). La frecuencia de consumo tiende hacia lo frecuente, ya que un $40.2 \%$ lo degusta varias veces por semana, el $19.1 \%$ una vez cada quince días y el $10 \%$ todos los días. Entre tanto el consumo mensual o más esporádico lo realiza el $30.8 \%$ de los consumidores.

Los atributos que más valoran las personas al momento de consumir un yogur (evaluados en una escala de cinco puntos) son: el sabor (4.50), seguido de la calidad (4.10) y la fecha de caducidad (3.91). Otros aspectos altamente apreciados son la cantidad (3.88), la marca (3.88) y el valor nutritivo (3.70). En cambio, aquellos que se valoran con puntuaciones menores tienen que ver con el efecto inmunológico (3.39), el envase (3.35), que sea enriquecido (3.33), el bajo contenido de calorías (3.28), que su producción sea orgánica (3.27) y el origen (2.70) (tabla 3).

Tabla 3. Valoraciones medias de atributos intrínsecos y extrínsecos del yogur

\begin{tabular}{|lc|lc|}
\hline \multicolumn{1}{|c|}{ Características $^{\text {a }}$} & Media & \multicolumn{1}{c|}{ Características $^{\text {a }}$} & Media \\
\hline Sabor & 4.50 & Diseño del envase & 3.47 \\
\hline Calidad & 4.10 & Control de peso & 3.46 \\
\hline Fecha de caducidad & 3.91 & Efecto inmunológico & 3.39 \\
\hline Cantidad & 3.88 & Envase & 3.35 \\
\hline Marca & 3.88 & Enriquecido & 3.33 \\
\hline Valor nutritivo & 3.70 & Bajo en calorías & 3.28 \\
\hline Envase cómodo & 3.58 & Producción orgánica & 3.27 \\
\hline Alto contenido en fibra & 3.53 & Origen & 2.70 \\
\cline { 1 - 2 } Presentación & 3.49 & \multicolumn{2}{|l}{} \\
\cline { 1 - 2 } & & &
\end{tabular}

${ }^{a}$ Los valores fueron medidos en una escala de 1 a 5 donde 1 =Nada importante y $5=$ Muy importante. Fuente: Elaboración propia.

Con respecto a los cambios que se realizan en el yogur y la valoración que otorgan los consumidores, se observa que los valores medios (cuantificados en una escala de cinco puntos) más altos correspondan a 
cambios en el precio (3.93), esto deja entrever una alta sensibilidad en su disposición a pagar. También resultan muy importantes los cambios que se efectúan en la facilidad de consumo (3.70), es decir: una modificación, por ejemplo, de yogur sólido a líquido. Otros aspectos con una alta valoración son las variaciones en los ingredientes (3.69), así como el mayor tiempo de caducidad (3.59). Al contrario: el cambio en la información nutrimental (3.52), cambio en la disponibilidad de establecimientos (3.37), cambios en la presentación (3.23) y cambios en el diseño del envase (2.93) son los que menor grado de importancia tienen para el consumidor (tabla 4). En general, por las valoraciones obtenidas por encima de la media de la escala, se percibe que los consumidores muestran una actitud favorable a las innovaciones que se realizan en el yogur, incluso acentuando su importancia en algunos atributos y características como el precio, facilidad en su consumo e ingredientes.

Tabla 4. Valores medios en relación a las innovaciones en el yogur

\begin{tabular}{|lcc|}
\hline \multicolumn{1}{|c|}{ Mejora/cambio $^{\text {a }}$} & Media & Desviación típica \\
\hline Cambio en el precio & 3.93 & 1.08 \\
\hline Cambio en la facilidad de consumo & 3.70 & 1.19 \\
\hline Cambio en los ingredientes & 3.69 & 1.27 \\
\hline Mayor tiempo de caducidad & 3.59 & 1.22 \\
\hline Cambio en la información nutrimental & 3.52 & 1.28 \\
\hline Disponibilidad de establecimientos & 3.37 & 1.21 \\
\hline Cambio en la presentación & 3.23 & 1.30 \\
\hline Cambio en el diseño del envase & 2.93 & Muy importante \\
\hline
\end{tabular}

Fuente: elaboración propia.

Considerando las variables anteriores (tabla 4), se realizó la prueba de fiabilidad de la escala, con la cual se obtuvo un alfa de Cronbach de 0.859 , valor que demuestra que existe consistencia interna en la escala. A partir de las puntuaciones individuales se procedió, en una primera etapa, a realizar un análisis factorial para condensar la información original en un número inferior de variables o factores. Las puntuaciones factoriales obtenidas para cada individuo se guardan y se utilizan, en una segunda etapa, como bases de segmentación del análisis clúster. Una vez clasificados los consumidores en los segmentos o clúster definidos, se procede a describir sus perfiles, en función de las variables socioeconómicas no implicadas en la segmentación, como edad, género, entre otras.

Los valores se agruparon en cuatro factores que explican el $79.22 \%$ de la variabilidad de los datos, valor que de acuerdo con Hair, Black, Babin, Anderson \& Thatam (2005) se considera admisible. El primer factor explica el $50.47 \%$ de la varianza total y está relacionado con cambios en el consumo, 
información nutrimental y precio. De ahí que a este factor se le ha denominado información y consumo. El segundo factor recoge el $13.5 \%$ de la varianza total y se integra por modificaciones en presentación, diseño y disponibilidad; por tanto, se le ha llamado diseño. El tercer y cuarto factor explican el $8.56 \%$ y $6.73 \%$ de la varianza total, respectivamente. Se integran por una variable, por lo que se han designado como ingredientes y caducidad.

Tabla 5. Análisis factorial de los cambios/innovaciones en el yogur

\begin{tabular}{|l|c|c|c|c|c|}
\hline \multicolumn{1}{|c|}{ Variable } & Comunalidad & Factor 1 & Factor 2 & Factor 3 & Factor 4 \\
\hline Cambio en la facilidad de consumo & .708 & $\mathbf{. 7 7 0}$ & .235 & .152 & .188 \\
\hline $\begin{array}{l}\text { Cambios en la información } \\
\text { nutrimental }\end{array}$ & .800 &. $\mathbf{7 5 5}$ & .120 & .462 & .038 \\
\hline Cambio en el precio & .737 & $\mathbf{. 7 4 7}$ & .182 & -.087 & .374 \\
\hline Cambio en la presentación & .857 & .298 & $\mathbf{. 8 7 5}$ & .019 & .034 \\
\hline Cambio en el diseño del envase & .764 & .045 & $\mathbf{. 7 8 6}$ & .355 & .135 \\
\hline Disponibilidad de establecimientos & .692 & .279 & $\mathbf{. 6 0 5}$ & .325 & .377 \\
\hline Cambio en los ingredientes & .875 & .172 & .310 & $\mathbf{. 8 3 3}$ & .236 \\
\hline Mayor tiempo de caducidad & .905 & .297 & .147 & .228 & $\mathbf{. 8 6 2}$ \\
\hline $\begin{array}{l}\text { Varianza explicada (\%) } \\
\text { Varianza total explicada (\%): 79.22 } \\
\alpha \text { de Cronbach: 0.859; test de esfericidad de Bartlett: 701.569 (valor p: } 0.000)\end{array}$ \\
Medida de adecuación muestral de Kaiser-Mayer-Olkin (KMO): 0.853 \\
\hline
\end{tabular}

Método de extracción: análisis de componentes principales. Método de rotación: normalización Varimax con Kaiser. La rotación ha convergido en 7 iteraciones. En negrita, se señalan las correlaciones más elevadas dentro de cada factor.

Fuente: elaboración propia.

A partir de las puntuaciones factoriales obtenidas a análisis factorial aplicado a las innovaciones hacia el yogur, se aplicó la técnica k-means de análisis clúster, con el fin de obtener grupos que aglutinen a consumidores con comportamientos semejantes (tabla 6). Los resultados han permitido clasificar a los consumidores en dos grupos: en el primero se encuentran aquellos que conceden mayor importancia al diseño e información del producto, mientras que en el segundo grupo se ubican aquellas personas que dan especial atención a toda la información relativa a caducidad e ingredientes (tabla 6). Los dos segmentos ]L 0987son distintos en tamaño: el primero representa al 55.8\% y el segundo, al $44.1 \%$ de la muestra. Al primer segmento se le denomina: consumidores orientados al diseño y consumo y al segundo, consumidores orientados hacia sabor y seguridad. Seguidamente, considerando la segmentación de los consumidores en función de su valoración hacia las innovaciones, se procedieron a realizar los correspondientes análisis de test de chi-cuadrado. En la tabla 7 se muestran las variables que obtuvieron asociaciones significativas. 


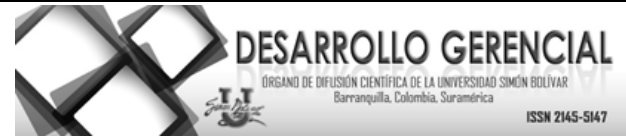

Tabla 6. Segmentación de los consumidores en función de su valoración hacia las innovaciones en el yogur

\begin{tabular}{|l|c|c|c|}
\hline \multirow{2}{*}{} & \multicolumn{2}{|c|}{$\begin{array}{c}\text { Conglomerado de } \\
\text { pertenencia }\end{array}$} & \multirow{2}{*}{ Sig. } \\
\cline { 2 - 3 } & $\mathbf{1}$ & $\mathbf{2}$ & \\
\hline Información y consumo & 0.575 & -0.729 & 0.000 \\
\hline Diseño & 0.372 & -0.472 & 0.000 \\
\hline Ingredientes & -0.139 & 0.177 & 0.017 \\
\hline Caducidad & -0.261 & 0.273 & 0.000 \\
\hline \% de personas & 55.8 & 44.1 & \\
\hline
\end{tabular}

Fuente: elaboración propia.

A continuación se trata de describir el perfil sociodemográfico de los consumidores, aplicando el test de chi-cuadrado para contrastar si existen diferencias significativas entre los segmentos uno y dos de la tabla 6. Los resultados muestran que existen pocas variables que se diferencien estadísticamente entre segmentos. De acuerdo a los resultados obtenidos, se observa una asociación positiva con el sexo y la orientación de las innovaciones que se realizan en el yogur, por lo tanto, existe una mayor proporción de hombres que prefieren innovaciones en diseño y forma de consumo (46.9\%), mientras que en el caso de las mujeres la proporción es ligeramente mayor en aquellas que se orientan más hacia las innovaciones en sabor y seguridad (53.5\% frente a 53.1\%). De igual manera existen diferencias significativas entre la edad y la importancia que los consumidores otorgan a las innovaciones en el yogur. Así, los consumidores en el rango de 18 a 32 y los mayores de 48 años de edad, tienden más hacia las innovaciones en sabor y seguridad, mientras que aquellos que tienen entre 33 y 47 años se orientan más hacia las de diseño.

Posiblemente, los consumidores más jóvenes valoran con preferencia las innovaciones en sabor, mientras que los mayores se orientan prioritariamente hacia las de seguridad, por cuestiones de cuidado a la salud. Por último, en lo que respecta al nivel de estudio y la valoración de los consumidores hacia las innovaciones también se identifican diferencias significativas. Los resultados dejan entrever que a medida que el nivel educativo de las personas se incrementa, su orientación hacia las innovaciones tiende más hacia el sabor y la seguridad.

Tabla 7. Perfil de los segmentos obtenidos en función de su orientación en la innovación

\begin{tabular}{|c|c|c|c|}
\hline \multirow{2}{*}{$\begin{array}{l}\text { Segmentación } \backslash \\
\text { Variables }\end{array}$} & \multirow{2}{*}{$\begin{array}{c}\text { Test } \\
\frac{\text { Chi- cuadrado }}{\mathrm{X}^{2}} \\
\text { (valor de prob.) }\end{array}$} & \multicolumn{2}{|c|}{$\begin{array}{l}\text { Consumidores orientados a valorar más innovaciones en: } \\
\text { (porcentaje de consumidores dentro de cada segmento) }\end{array}$} \\
\hline & & Diseño y consumo $(55.8 \%)$ & $\begin{array}{l}\text { Sabor y seguridad } \\
(44.1 \%)\end{array}$ \\
\hline Hombre & \multirow{2}{*}{$0.003 *$} & 46.9 & 46.5 \\
\hline Mujer & & 53.1 & 53.5 \\
\hline 18-22 años & \multirow{3}{*}{$0.070 * *$} & 11.7 & 16.8 \\
\hline 23-27 años & & 16.4 & 20.8 \\
\hline 28-32 años & & 11.7 & 12.9 \\
\hline
\end{tabular}




\begin{tabular}{|c|c|c|c|}
\hline 33-37 años & & 14.8 & 7.9 \\
\hline 38-42 años & & 10.9 & 6.9 \\
\hline 43-47 años & & 11.7 & 7.9 \\
\hline 48-52 años & & 8.6 & 9.9 \\
\hline 53-57 años & & 3.9 & 5.9 \\
\hline 58-62 años & & 1.6 & 6.9 \\
\hline 63 o más años & & 4.0 & 8.6 \\
\hline Sin estudios & \multirow{8}{*}{$0.000 *$} & 1.6 & 1.0 \\
\hline Primaria & & 2.3 & 1.0 \\
\hline Secundaria & & 11.7 & 7.9 \\
\hline Preparatoria & & 16.4 & 14.9 \\
\hline Carrera técnica & & 14.8 & 19.8 \\
\hline Universitarios & & 45.3 & 45.5 \\
\hline Maestría & & 7.8 & 7.9 \\
\hline Doctorado & & 0.0 & 2.0 \\
\hline
\end{tabular}

Fuente: elaboración propia.

\section{CONCLUSIONES}

Las innovaciones se han posicionado como importantes estrategias en el ámbito empresarial, pues tienen que ver con la introducción de nuevos productos y servicios, con nuevos procesos, nuevas fuentes de abastecimiento y cambios en la organización industrial, en forma continua, y orientadas al consumidor o usuario (Schumpeter, 1934). La influencia e impacto que las innovaciones ejercen en las empresas ha llevado a su análisis desde distintos enfoques, de los cuales uno se centra en la demanda final.

Desde esta perspectiva, la presente investigación aporta directrices para la comprensión y conocimiento de las actitudes de los consumidores del noroeste de México hacia las innovaciones en el yogur. En este sentido, se advierte que en el mercado se comercializan ocho marcas de yogur, que en todos los casos se diferencian en las presentaciones, diseños y sabores. Esta situación evidencia el uso de las innovaciones como una herramienta de diferenciación empresarial, tal y como han encontrado otros autores (Drucker, 1985; Tushman \& Nadler, 1986).

Por otro lado, se identificó que las innovaciones en el producto se concentran en aspectos de diseño y de sabor, que en la mayor parte de los casos refiere a innovaciones incrementales y de atributos extrínsecos; estos resultados coinciden con los de Mueller et al. (2013). Del mismo modo, quedó patente que las preferencias de los consumidores tienden hacia sabores tradicionales.

Ahondando un poco más en el análisis, se reconoció que los consumidores se pueden agrupar en aquellos que valoran las innovaciones en el diseño y practicidad en su consumo, frente a los que aprecian el sabor y la seguridad. Pero en los primeros se concentra una mayor proporción de consumidores. Estos 


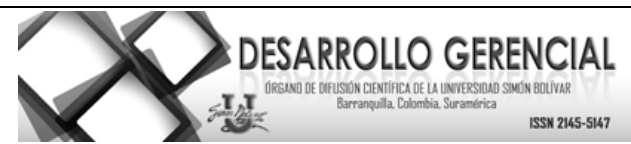

resultados son similares a los encontrados en la literatura, particularmente con ciertos productos como los ostiones, donde atributos de diseño, presentación y empaque son apreciados por los consumidores (Mueller, Peschel \& Grebitus, 2013). Sin embargo, son contrarios a los obtenidos en otros productos lácteos como el queso, donde los aspectos de proceso tienen mayor peso entre los consumidores (Lengard, Naes, Enderli, Sulmont-Rossé, Issanchou \& Hersleth, 2011). Básicamente, la valoración de los atributos en las innovaciones no se relaciona con categorías de alimentos, sino con productos en particular, por lo que es importante cuidar ese detalle al momento de estudiar comidas o alimentos.

También se ha visto que las características sociodemográficas como la edad y el nivel educativo, influyen en las actitudes de los consumidores, tal y como encontró Barrena et al. 2013. A mayor edad y nivel de estudios, el riesgo percibido y el cuidado a la salud toman mayor relevancia; de ahí que una valoración hacia aspectos de seguridad sea más acentuada. Estos resultados son coincidentes con los encontrados por Pilone et al. (2014), donde elementos de confianza y prolongación de vida en anaquel tienen un peso importante en las decisiones de los consumidores italianos.

En términos generales, los resultados aquí obtenidos representan un avance en el conocimiento del consumidor ante las innovaciones. A partir del análisis efectuado es posible identificar segmentos de mercados y la valoración que realizan hacia los distintos atributos del yogur. Para el empresario, la información resulta útil al momento de considerar estrategias de posicionamiento de su producto o de decidir el tipo de innovaciones que va a efectuar de cara a una mejor comercialización. Así, una adecuada estrategia de innovaciones puede direccionarse en satisfacer la demanda de acuerdo a las características que más se valoran, por ejemplo, personas mayores de 48 años de edad apreciarán más innovaciones en seguridad, calidad en los ingredientes y beneficios a la salud. Entre tanto, aquellas más jóvenes apreciarán aspectos relacionados con el diseño y presentación.

\section{REFERENCIAS}

Alvarado, E., Luyando, J. \& Téllez, R. (2012). Caracterización del consumidor de la carne de pollo en el área metropolitana de Monterrey. Región y Sociedad, 24 (54), 175-199.

Arcas, L. (2003). Contribución de las cooperativas agrarias de segundo grado a la orientación al mercado de sus cooperativas asociadas: efectos en el desempeño de la relación Ciriec-España. Revista de Economía Pública, Social y Cooperativa, 41, 139-161.

Barrena, R., García, T., \& López-Mosquera, N. (2013). Determinantes socioeconómicos y emocionales en el consumo de nuevos alimentos. España: Universidad Pública de Navarra. 


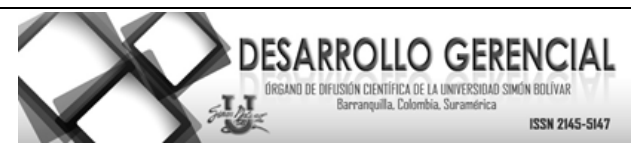

Briones, C. (2005). Proceso de elaboración de yogur y su presentación en página web. Monografía, Universidad Autónoma del Estado de Hidalgo.

Camarena, D., Sanjuán, A.I. \& Philippidis, G. (2011). Influence of ethnocentrism and neo-phobia on ethnic food consumption in Spain. Appetite, 57, 121-130.

Camarena, D. \& Sanjuán, A.I. (2006). Consumer preferences for walnuts in Spain. Journal of Food Products Marketing, 12 (4), 39-57.

Cortés, G.; Mora, J.; García-Mata, R. \& Ramírez-Valverde, G. (2012). Estudio del consumo de la carne de cerdo en la zona metropolitana del Valle de México, Estudios Sociales. Revista de Investigación Científica, 20 (40), 335-352.

Díaz, M., Prieto, A., \& Bernabéu, R. (2013). Estructura de las preferencias de los consumidores de carne de cordero en Castilla-La Mancha. ITEA, 109 (4), 476-491.

Díaz de Rada, V. (1998). Diseño de tipologías de consumidores mediante la utilización conjunta del Análisis Cluster y otras técnicas multivariantes. Economía Agraria, 182, 75-104.

Drucker, P. (1985). Innovation and entrepreneurship. New York: Harper Row Publishing.

Earle, M.D. (1997). Innovation in the food industry. Trends in Food Science \& Technology, 8, 166-175.

Fernández, A. (2004). Investigación y técnicas de mercado (2. ${ }^{a}$ ed.). Madrid: ESIC Editorial.

Hair, J.F., Black, W., Babin, B., Anderson, R.E., \& Tatham, R.L. (2005). Multivariate data analysis (5th ed.). Upper Saddle River, NJ: Prentice Hall.

Hernández, R., Fernández, C., \& Baptista, P. (2010). Metodología de la investigación. México: McGrawHill.

Hoban, T. J. (1998). Improving the success of new product development. Food Technology, 52(1), 46-49.

Instituto Nacional de Estadística y Geografía INEGI (2010). Censo de población y vivienda. Recuperado de: http://www.inegi.org.mx/

Lengard, V., Naes, T., Enderli, G., Sulmont-Rossé, C., Issanchou, S. \& Hersleth, M. (2011). Consumers' acceptance of innovations in traditional cheese. A comparative study in France and Norway. Appetite, 57, 110-120.

Montoya, O. (2007). Aplicación del análisis factorial a la investigación de mercados. Caso de estudio. Scientia et Technica, 35, 281-286. 


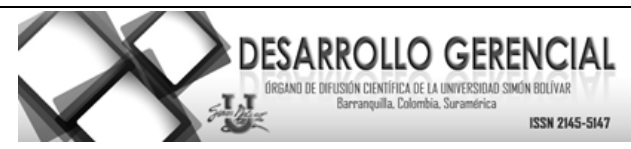

Mueller, S., Peschel, A. \& Grebitus, C. (2013). Quantifying effects of convenience and product packaging on consumer preferences and market share of seafood products: The case of oysters. Food Quality and Preference, 28 (2), 492-504.

OCDE. (2005). Manual de Oslo ( $3^{\mathrm{a}}$ ed.). Francia: Autor y Eurostat.

Pilone, V., De Lucia, C., Del Nobile, M.A. \& Contò, F. (2014). Policy developments of consumer's acceptance of traditional products innovation: The case of environmental sustainability and shelf life extension of a PGI Italian cheese. Trends in Food Science \& Technology, 1-12.

Ramírez, D. \& Jiménez, W. (2013). Percepción de riesgo y compras por internet: Su relación con la personalidad y el tipo de producto. Suma Psicológica, 20 (2), 147-161.

Ritchey, F. (2002). Estadística para las Ciencias Sociales. México: McGraw-Hill.

Salcedo, C., Font, M. \& Martínez, R. (1988). Yogur. Elaboración y valor nutritivo, Divulgación (10), 343.

Schumpeter, J. (1934). The Theory of Economic Development. Cambridge: Harvard University Press.

Siriwongwilaichat, P. (2001). Technical information capture for food product innovation in Thailand. Ph. D. Thesis. Massey University of New Zealand.

Tushman, M. \& Nadler, D. (1986). Organizing for innovation. California Management Review, 28(3), 7492.

Winger, R. \& Wall, G. (2006). Food product innovation: A background paper. Rome: Food and Agriculture Organization. 